\title{
Mujer indígena: presencia (in)visible en la representación social y cultural de la población indígena mexicana Estefanía Soto Carrasquel
}

Egresada del Colegio del Estado de Hidalgo,mpdr0617@elcolegiodehidalgo.edu.mx Recibido: 15-11-18 Aceptado: 19-02-2019 Publicado: 15-08-2019

Cómo citar este artículo: Soto E. (2019) "Mujer indígena: presencia (in)visible en la representación social y cultural de la población indígena mexicanana" en Revista Científica de Estudios Urbano Regionales Hatsö-Hnini, Año 1, Núm. 1, Abril-Septiembre 2019, págs. 72-82 México

DOI: https://doi.org/10.47386/2019V1N1A6

Se ha escrito bastante ya sobre feminismo y arte, sobre historia y teoría feminista del arte, pero, curiosamente, el pensamiento feminista de los países desarrollados, que es el que se ha ocupado más de investigar en el campo del arte, le ha interesado poco incursionar en el arte

popular; éste no entra dentro de la Historia del Arte, con mayúscula" Eli Bartra

\section{Resumen}

La definición de población indígena ha estado sujeta a diversos criterios e infinidad de debates; esta misma presenta un espectro de variación y contraste tan amplio que ninguna definición, a partir de sus características internas, puede incorporar a todas. En tanto, la socialización de los individuos desde el atisbo de la gentrificación de la población y cultura indígena sugiere que en el traspaso cultural de saberes se permite establecer y transmitir valores, normas y costumbres, actividad predominantemente ejercida por mujeres; sobre todo desde la concepción del arte popular textil, que es quizá uno de los pilares más importantes en la conformación identitaria nacional.

Palabras claves: población indígena, mujer, arte popular, arte textil, hogar, pertenencia, identidad.

\section{Introducción}

El casi nulo o escaso interés que se le tiene al arte popular y su proceso creativo ha sido la causa de que no se efectúen investigaciones formales sobre él y sobre todo ha provocado que se deje de lado a - los creadores, personas que actúan de manera invisible, que por lo general son las mujeres, mujeres indígenas, sobre todo. ¿Es posible concebir y estudiar al arte popular indígena desde el punto de vista de la división genérica, haciendo particular énfasis en la mujer indígena para conocer la estructura social y la construcción cultural de la población indígena?

En México la población indígena tiende a formar parte de los estratos sociales más pobres y desfavorecidos; "con frecuencia, sus niveles de vida están por debajo de los promedios nacionales y regionales, así como de los mínimos de bienestar establecidos internacionalmente. Pero el hecho de que compartan las mismas características y carencias generales no significa que todos los pueblos indígenas y todos los indígenas sean iguales; por ello es importante conocer sus diferencias" (García, et al, 2006).

\section{Desarrollo y discusión}

La definición de población indígena ha estado sujeta a diversos criterios e infinidad de debates desde la mirada antropológica, sociológica y demográfica. Uno de ellos, y quizá el que ha estado con mayor frecuencia en el centro del debate, es el criterio de la lengua hablada por los individuos, que ha sido y es la base para la recopilación de información en los censos de población y encuestas; sin embargo, durante el - 
proceso de conformación de la nación la imposición de una lengua nacional, es una medida homogeneizadora que buscaba la abolición de la identidades étnicas, y por tanto forma parte de un proceso de deformación de las culturas cuya misión civilizadora subordinaba todo particularismo que pudiera oponerse a los fines de la expansión capitalista y constituía el fundamento de las nuevas formas de dominación, situación que lleva a pensar si ¿la definición de población indígena puede ser dada a partir de una sola variable referida por la cuantificación de una dimensión subjetiva (el lenguaje) de la cultura de ésta población?

En este mismo esquema, las variables incluidas en los Censos de Población y Vivienda que presenta el Instituto Nacional de Estadística, Geografía e Informática (INEGI) incluyen a personas de 5 años y más, hablantes de lengua indígena, sin embargo esta medida tiende a excluir a la población infantil indígena y a aquellos que no hablan una lengua indígena: por tal motivo se proponen otros criterios de aproximación, como el hogar, el cual permite conformar un conjunto demográficamente coherente y aprehender de las relaciones socioculturales que identifican a los individuos como parte de un sistema de creencias, valores, normas, símbolos y prácticas colectivas aprendidas por los miembros de una colectividad y que constituyen el marco de referencia para la construcción de las relaciones sociales. Solo entonces se puede incluir al hogar indígena como una variable más para la cuantificación de la población indígena, definiéndolo a manera de "aquel en donde el jefe del hogar o alguno de sus parientes habla lengua indígena o pertenece a un grupo indígena, en donde, podemos incluir una variable más: la pertenencia; que expresa la existencia de criterios simbólicos de conducta y la herencia cultural en el interior del hogar más allá del criterio lingüístico, por lo que la inclusión permite identificar con mayor amplitud a 1 a p ob la c i ón i n díg e na, independientemente de si hablan o no una lengua indígena" (García, et al, 2006).

Parafraseando a Bonfil Batalla, en su texto "El concepto del indio en América: una categoría de la situación colonial" (1972) la definición de indígena no puede basarse en el análisis de las particularidades propias de cada grupo y las sociedades; la cultura llamada indígena presenta un espectro de variación y contraste tan amplio que ninguna definición a partir de sus características internas puede incorporarse a todas, so pena de perder cualquier valor heurístico. Son indígenas quienes poseen el dominio de características de cultura material y espiritual peculiares, distintas de las que hemos dado en llamar "Cultura occidental".

La Comisión Nacional para el Desarrollo de los Pueblos Indígenas, desarrolló una tipología que ayuda a identificar la distribución de la población indígena en el territorio nacional, "de esta manera se ha considerado un criterio basado en la territorialidad, que define localidades y municipios como indígenas y no indígenas de acuerdo al volumen o porcentaje de población indígena en cada uno de ellos" (García, et al, 2006), de acuerdo con esta definición y clasificación establecida por la Comisión Nacional para el Desarrollo - 
los Pueblos Indígenas se consideran:

a. Municipio indígena: 40 por ciento y más de la población en hogares indígenas.

b. Municipios con presencia indígena: menos del 40 por ciento en hogares indígenas, pero con un volumen de 5 mil y más indígenas y/o con población indígena de lenguas minoritarias.

c. Municipios con población indígena dispersa: menos de 40 por ciento y menos de 5 mil indígenas.

En cuanto a las localidades, se consideran indígenas aquellas en donde 40 por ciento o más de sus habitantes son indígenas; por su concentración, se divide en dos grupos:

a. De 40 a 69 por ciento de población en hogares indígenas

b. De 70 por ciento y más de población en hogares indígenas.

En México la población indígena asciende a un total de 12 millones 25 mil 947 personas, que representa el $10.1 \%$ de la población nacional. Del total de población indígena estimada 6 millones 146 mil 479 son mujeres, población que representa el 51.1\%; mientras que la población indígena masculina es de 5 millones 879 mil 468, es decir el 48.9\%.

Como se muestra en el Cuadro 1, la distribución de la población indígena a nivel nacional se diferencia ocho entidades federativas las que concentran la mayor población:

Oaxaca y Chiapas con 1.7 millones cada una, y son las que cuentan con la mayor cantidad de población indígena equivalente al $14.4 \%$ y $14.2 \%$ respectivamente, a. Veracruz con 1.1 millones de población indígena que equivale al $9.2 \%$, b. México un estimado de población indígena de 1.097 millones que representa el $9.1 \%$,

c. Puebla con 1.094 millones, aproximadamente el $9.1 \%$,

d. Yucatán cuya población indígena se estima en 1.052 millones, es decir, $8.8 \%$,

e. Guerrero con 681 mil 615, solo el $5.7 \%$,

f. Hidalgo 606 mil 45, el 5.0\%.

En el caso contrario, solo se muestran seis entidades del territorio nacional cuya población indígena total suma menos de 30 mil personas:

a. Guanajuato con 29 mil 863,

b. Baja California Sur cuya población asciende a 23 mil 456 ,

c. Coahuila con 13 mil 349,

d. Colima con un total de 10 mil 122,

e. Aguascalientes con 9 mil 306,

f. Zacatecas con tan solo 7 mil 852.

Gracias a esta tipología se puede considerar, que los municipios indígenas son aquellos en donde $40 \%$ o más de su población total indígena, suman 623 y en ellos se concentra el $56.4 \%$ de la población indígena; distribución observada en el Mapa 1 (pág.8). En estos municipios viven 3 millones 488 mil 713 mujeres indígenas, que representa $56.8 \%$ y 3 millones 295 mil 79 hombres, $56.1 \%$ (CDI, 2015). 
Cuadro 1.- Distribución de población indígena en México POBLACIÓN INDÍGENA POR SEXO Y ENTIDAD FEDERATIVA, 2015

Mujer $\square$ Hombre

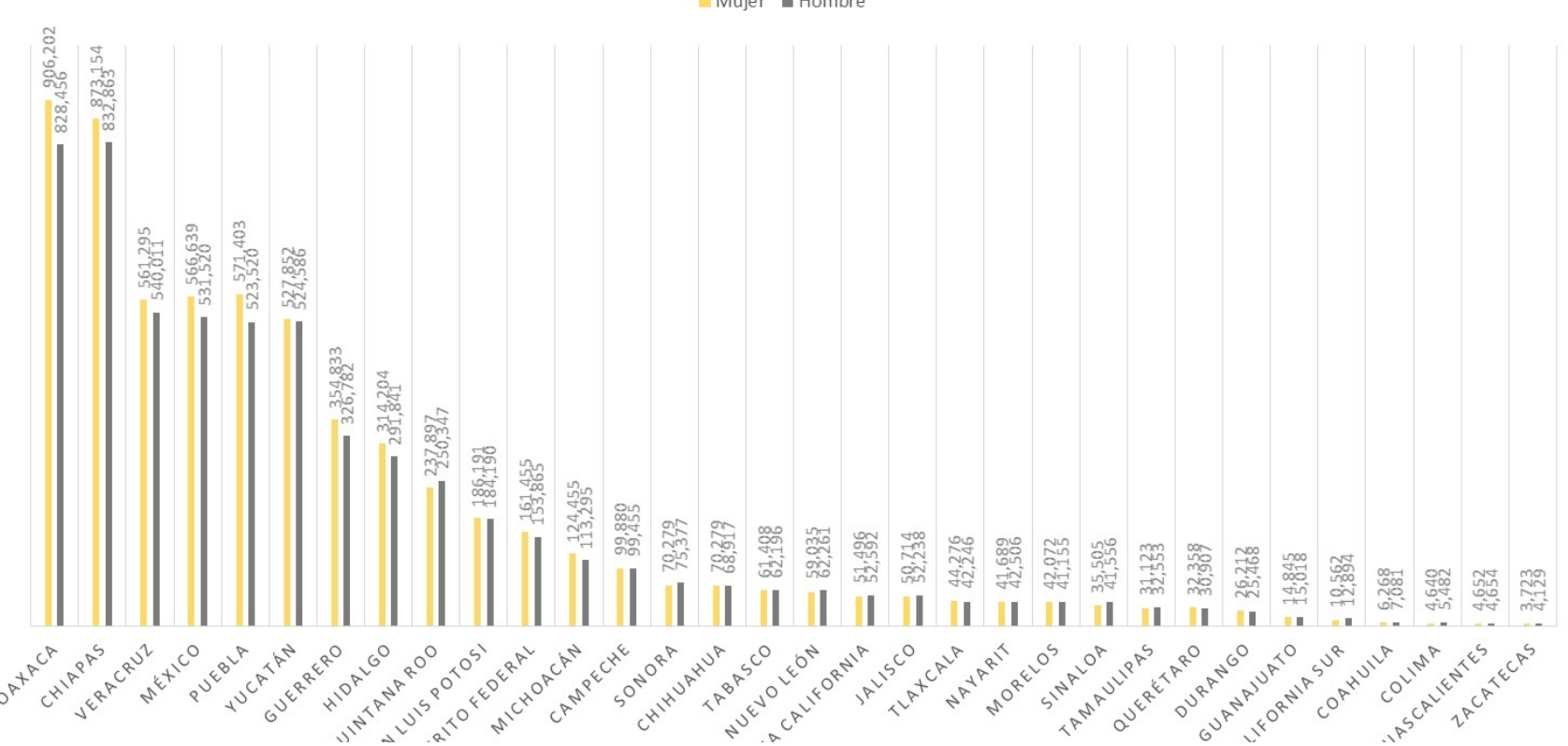

Fuente: Comisión Nacional para el Desarrollo de los Pueblos Indígenas, Encuesta Intercensal, INEGI, 2015.

Figura 1.- Localidades con $40 \%$ y más de población indígena

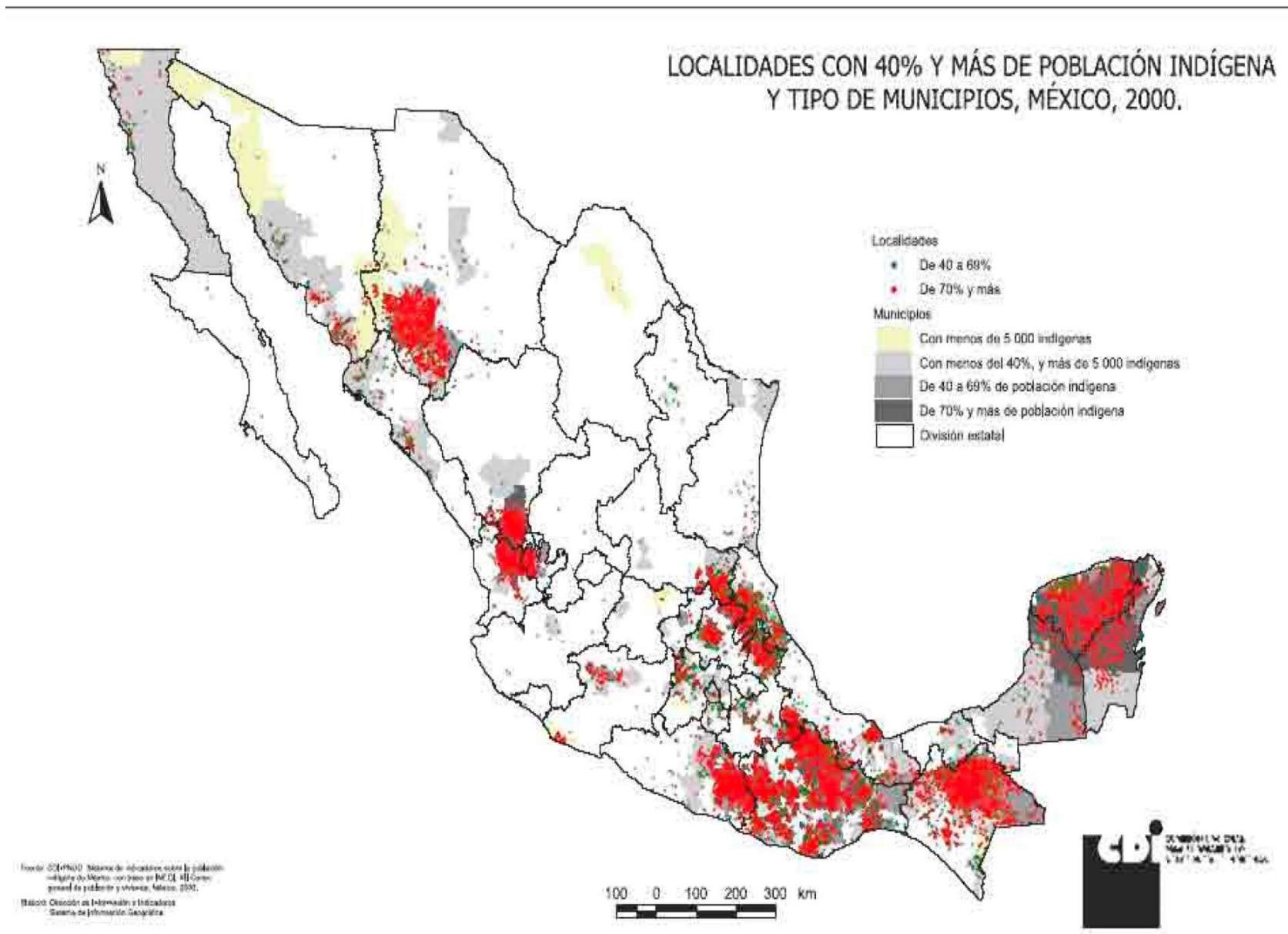

Fuente: Comisión Nacional para el Desarrollo de los Pueblos Indígenas, 2000 


\section{Mujer indígena: presencia (invisible) en la representación social y cultural}

Como se ha dicho con anterioridad, para la identificación de la población indígena se deben considerar diferentes criterios, se sugiere entonces al hogar como uno de ellos, puesto que se concibe como un espacio de identificación y transmisión de la cultura; dentro de él existen lazos de parentesco y afectividad fuertes que inciden en el desarrollo de las identidades. El papel del hogar en la socialización de los individuos y en la transmisión cultural permite suponer que en aquellos hogares en los que las generaciones encargadas de establecer y transmitir valores, normas y costumbres son personas indígenas, en su mayoría mujeres.

La realidad demográfica y social de nuestro país, sobre todo hablando en términos de población indígena y de la condición de la mujer dentro de ese contexto, "hace evidente el condicionamiento social de género, que afecta negativamente a las mujeres en aspectos tales como su participación en el mercado del trabajo y su acceso a los beneficios de algunas políticas públicas orientadas al alivio de la pobreza" (Acosta, 1995). Todo esto se presenta a pesar de que la participación de la mujer indígena se encuentra intrínsecamente ligada al contexto de las dinámicas familiares y está hace palpable su creciente contribución a la generación de ingresos.

Detrás de este proceso de hacer manifiesta la incorporación de la mujer a las actividades extra domésticas, se encuentra el deterioro de los niveles de vida de las familias mexicanas de menores ingresos; que como señala Félix Acosta en su texto "Participación femenina, estrategias familiares de vida y jefatura femenina de hogar: los problemas de la jefatura declarada" (1995) es "producto de la caída acumulada durante veinte años de los ingresos reales y debido a la escasez de empleo masculino. La existencia de estos procesos sugiere que la responsabilidad económica de la mujer en los hogares, especialmente los de bajos ingresos, se ha incrementado" (Acosta, 1995).

Sin embargo, la preocupación por los problemas de los grupos étnicos no es un tema reciente; por ejemplo, el debate sobre los criterios que definen la identidad indígena ha existido por un largo periodo, uno de los principales ejes de este debate es la cuestión que comprende los procesos de aculturación e hibridación cultural, así como la integración social, entendida como un proceso de homogeneización étnica y cultural de la población, ha sido la fuerza motriz de la "construcción nacional".

"El respeto a la identidad cultural de los pueblos no puede ser un mero derecho pasivo, no se puede negar a las personas que pertenezcan a las minorías étnicas el derecho que les corresponde a tener su propia vida cultural y a emplear su propio idioma. El respeto a la identidad cultural de los pueblos debe ser un derecho activo que se traduzca en políticas de apoyo al desarrollo cultural de las minorías" (Stavenhagen, 1984).

Por lo tanto, la diversidad cultural y la heterogeneidad en las formas de vida presentes en una sociedad, como la mexicana, obligan a la inclusión de la opinión de los distintos grupos y sectores que la componen. 


\section{Mujer indígena: presencia (invisible) en la representación social y cultural}

A su vez, "como parte de la instrumentación, seguimiento y evaluación de las políticas y acciones institucionales, es requisito contar con un referente de la situación de la mujer en contextos particulares y específicos, es decir, acordes con esta realidad, contextuar a la mujer indígena en su entorno sociocultural, y establecer una dimensión" (García, et al, 2006) en donde las desigualdades por condición de género obedezcan a la persistencia de patrones culturales que se expresan en comportamientos de trascendencia primordial en el curso de la vida de las personas y que, si bien no son exclusivas de los grupos indígenas, sitúan a las mujeres indígenas en mayor desventaja social.

Si bien, la población femenina es la que presenta mayores rezagos, tanto en términos absolutos como relativos el trabajo de la mujer reviste particular interés, ya que se ha concebido una idea errónea respecto a su participación en la producción y su papel en la supervivencia de la familia. La inserción de las mujeres en la vida social y económica del espacio indígena suele estar subregistrada; gran parte de las tareas que realizan están bajo una organización comunitaria y familiar, en la que se considera "ayuda"; sin embargo, tener conocimiento de lo que la mujer indígena ha creado y crea hoy en día, ayuda sustancialmente para enas res.n personas indígenas, en su mayorde ellasentender mejor al grupo social. Saber en qué medida su proceso es igual o diferente al del hombre puede contribuir tanto a la formación de una identidad femenina más integral como a cambiar las condiciones de su existencia; en la medida en que se revalore el trabajo femenino avanzará la recuperación de una historia ignorada y el reconocimiento de que una parte de la cultura presente es suya.
La presencia femenina en torno a la cultura de los pueblos indígenas y sobre todo en la creación del arte, es de suma relevancia dada la tradición histórica de trasmitir de generación en generación los saberes y conocimientos de los antepasados, la mujer tiende a ser el pilar de las tradiciones; un ejemplo claro es el rol de la mujer en el arte popular, pero ¿de qué hablamos cuando nos referimos a Arte Popular? "El Arte Popular tiene su origen en la necesidad creadora del hombre; en la necesidad de producir objetos útiles y bellos" (Marín, 1976). Envolverse de lo placentero, de lo agradable, de lo útil y hasta indispensable es el estado natural del ser humano.

Lo bello, que es una interpretación subjetiva del individuo se manifiesta así individualmente; $y$ esta interpretación se materializa o se refleja a tenor de la capacidad creativa de cada quien [...] la capacidad creativa del individuo se mide en términos de sensibilidad, inteligencia, imaginación, cono-cimiento, inventiva, ingenio, experiencia, visión, educación, sentimiento, y la suma de todo ello constituye el grado mayor o menor de sabiduría con que realiza el objeto de su creación" (Marín, 1976).

Trasladar sensaciones y saberes a otros contextos culturales, desde el antiguo México prehispánico ha sido labor, principalmente, de la mujer, como afirma Irmgard Weitlander Jonhson (2005) "poco después del nacimiento de una niña, se le daban utensilios para hilar y tejer y simbólicamente se le instruía en su uso", el tejido entonces tiene una larga historia y trascendencia en la identidad de los pueblos, desde las 


\section{Mujer indígena: presencia (invisible) en la representación social y cultural}

culturas prehispánicas en donde, como ha sido mencionado, se desarrollaron técnicas de tejidos como parte de la manifestación cultural, relatando a través de símbolos su ideología ancestral, mística y religiosa.

El "arte popular es algo que ha resultado sumamente útil para consolidar una identidad cultural de carácter nacional en países plurietnicos, como el nuestro, pues se toman aspectos propios de cada cultura, en particular del arte y se amalgaman en una unidad identitaria nacional" (Bartra, 2015). Por ejemplo, en México después de la Revolución de 1910 , cuando era tan indispensable construir un Estado-nación, la utilización del arte popular conformaba la unicidad dentro de un universo étnico y culturalmente hablando era tan vasto.

En México, la gran abundancia de imaginería a través de la historia ha producido una gran mina de productoras de arte popular; productoras anónimas que sostienen, quizá, uno de los pilares más fuertes de la identidad nacional.

La participación femenina, así como la de los niños y las niñas, en el arte popular mexicano queda frecuentemente escudada detrás de la neutralidad del concepto de pueblo, por ello es preciso poner al descubierto su presencia en la creación, la distribución y el consumo del arte; "la inmensa mayoría de los trabajo sobre arte popular son en lo fundamental androcéntricos, producen entonces saberes sesgados por tratarse de un arte que hace el pueblo, en el cual solo hay hombres y las mujeres son invisibles, de ahí la necesidad de una labor de deconstrucción y reconstrucción" (Bartra, 2006).

El viajero, el turista, el antropólogo, atraviesan de norte a sur una nación en donde el arte popular es considerado artesanía, y los creadores artesanos, no - se consideran dentro de la Historia del Arte, "poco se ha incursionado en su espacio creativo, de producción y de consumo; en realidad lo que se manifiesta es una desvalorización deliberada o no, por supuesto, que al mismo tiempo, el arte popular es admirado, apreciado, consumido y usado, entusiasma y sorprende, pero de todas maneras es poco estudiado y mal valorado" (Bartra, 2006).

Por su parte, el arte textil en México ha alcanzado un alto valor a nivel de desarrollo, y es una labor que como pocas sobrevive en lo esencial entre algunos pueblos indígenas; muchas mujeres indígenas tejen y bordan, fijan la vista y mueven con precisión los brazos, las manos y los dedos para elaborar objetos delicados y útiles para la vida, objetos llamados de forma común artesanías. Llevan más de medio siglo dedicadas a combinar hilos de colores y realizar lienzos de diversos tamaños. Allí, en su patio acogedor hacen las tareas, preparan las madejas, mientras unas manejan con destreza la rueca o el malacate, otras hacen las putas, mientras se deshila un lienzo para después bordarlos, tiñen las fibras, recolectan materias primas para su fabricación, además enseñan a las más pequeñas cada uno de los elementos que conforman enseñanzas heredadas de generación en generación, mujeres que aprendieron en el seno familiar el arte del bordado y el tejido, en principio para elaborar su indumentaria y después para obtener ingresos económicos. El hilar, tejer y bordar en la historia se ha considerado una labor femenina, esta acción representa la creación y la vida, . 


\section{Mujer indígena: presencia (invisible) en la representación social y cultural}

entendida como multiplicación o crecimiento a partir de un hilo, el tejido, el oficio de tejer y los instrumentos para hacerlo, son símbolos que abren y cierran indefinidamente los ciclos originales de un pueblo.

Desde esta dimensión textil la mujer representa la amplitud y profundidad de la visión cultural, funge como el eslabón de los legados culturales más importantes de nuestro país, conocedora de técnicas del tejido, el bordado, así como su contexto etnográfico, abordando la memoria histórica que constituyen sus diseños y adentrándose en expresiones iconográficas significativas de cada una de las culturas que la mujer araña representa.

Asimismo, se debe tomar en cuenta que muchas de las familias de los pueblos indígenas complementan su ingreso con la producción artesanal, situación que coloca a la mujer como agente dominante en mayor o menor grado, como tutoras de la enseñanza y perfección de los conocimientos al utilizar sus habilidades para ayudar en la manutención de la familia. Justo en la búsqueda de mejores condiciones familiares se convierten en actor activo de comercialización y generan estrategias para proteger su cultura, y reivindican su participación como miembro activo de la sociedad. La importancia de traer esto a colación radica en conocer si realmente el rol de la mujer es visible para la estructura y contexto social en los que se desarrolla o es simplemente un actuar invisible.

Retomando a Eli Bartra en "Apuntes sobre feminismo y arte popular" (2015) hacer visible el rol femenino implica ver de cerca el quehacer de las mujeres incluidas sus segundas y terceras jornadas laborales.
Incorporar a su estudio la categoría de género no es algo frívolo, de moda o un capricho de algunas feministas, es un asunto nodal para la mejor comprensión de todo proceso, incluyendo el proceso artístico, significa profundizar, complejizar y obtener un conocimiento más cabal.

Lo que hay que evitar fervientemente es que las palabras se vuelvan simplemente una consigna discursiva de las políticas públicas, que sugieren "transversalizar la perspectiva de género" en todas partes. No se trata, pues, de eso, de transversalizar, sino de entender realmente la esencia de hablar en masculino y femenino, de comprender la existencia de dos o más géneros y lo que significa con respecto a lo que crean; advertir que esto tiene un peso específico para el desarrollo del conocimiento sobre el arte, ayuda a entender más a fondo todo lo referente al proceso de creación, de distribución, consumo, así como la iconografía de este mismo arte. Es importante saber si un objeto es creado por los huicholes, las purépechas, las mestizas, las zapotecas, las mayas o los chamulas; y si son ancianas, niñas, adultas; no es posible conocer a fondo este campo si se sigue pensando que el arte popular no tiene género y, por lo tanto, es producto de un pueblo abstracto y neutro" (Bartra, 2015).

El arte popular dado su carácter diferente, por su origen social, como por el proceso de creación, su distribución y su consumo, lo han convertido en un arte sin nombre, porque su creación se piensa como anónima o que tiene muchos nombres con ello se percibe que 


\section{Mujer indígena: presencia (invisible) en la representación social y cultural}

las mujeres indígenas, "las creadores y pilares del arte textil en México, y el arte popular tienen una relación símil pues están presentes en todo proceso social cotidiano pero son completamente invisibles, devaluados o poco respetados" (Bartra, 2015).

Entendiendo así que muchas de las tareas y labores de la mujer se señalan prácticamente invisibles, con frecuencia el arte popular es una de estas. "En la relación arte popular y las mujeres se entiende la inserción del actuar femenino en las dinámicas sociales y culturales de una comunidad, como elemento significativo del constructo identitario y de la activación económica, conocer la historia del bordado es conocer la historia de las mujeres" (Parker, 1996).

A pesar de recalcar en repetidas ocasiones que el arte popular es eje fundamental de la conformación de la identidad social y por ende las creadoras son agentes de cambio y continuidad, la mujer y el arte popular, siguen compartiendo una condición semejante: no obstante su presencia en la vida diaria, con mucha frecuencia son casi tan (in)visibles como insignificantes, además las características de los estudios sobre el arte popular muestran que la división genérica continúa siendo un hoyo negro; pues sucede periódicamente que "se escriben páginas y páginas sobre los objetos artísticos y no se menciona para nada a los o las creadores. Pero lo grave también es cuando sí se nombran y se pone de manifiesto un enorme desbalance entre hombres y mujeres" (Bartra, 2008).

\section{Conclusiones}

La cultura no es estática, y la mujer como agente activo es parte primordial de ese dinamismo. Advertir el rol de la mujer dentro de la dimensión del arte popular, específicamente desde la mirada del arte textil, no es mera cuestión de deporte intelectual, ni se trata de profundizar por el simple afán del conocimiento.

Resulta importante para el presente y el futuro de la mujer indígena, apuntar y recalcar la presencia de la mujer en las dinámicas sociales y sobre todo en espacios de creación, como el arte textil, determinantes de la identidad cultural de una sociedad, transformar lo (in) visible en visible. Situación que es importante pues debemos delimitar que hemos hecho y que estamos haciendo en cuanto a la definición de la identidad indígena y que tipo de identidades estamos construyendo.

Relacionar los diferentes criterios de adscripción étnica, la identificación y cuantificación de los grupos étnicos y pueblos indígenas en sociedades plurietnicas presenta un sinfín de dificultades metodológicas, desde plantear un nuevo enfoque conceptual que permita proyectar cuales son las áreas de intervención de una política en esta materia; por ejemplo, pasando de considerar aspectos exclusivamente económicos o de auto adscripción para darle un mayor peso a los aspectos culturales como la producción simbólica y material del arte popular, incluir la división genérica en éste con la intención de poner en manifiesto la existencia de un estado jerárquico y la necesidad de cambiarlo. 


\section{Mujer indígena: presencia (invisible) en la representación social y cultural}

Figura No. 2

San Pedro Sochiapam, Región de la Cañada, Oaxaca, 2016.

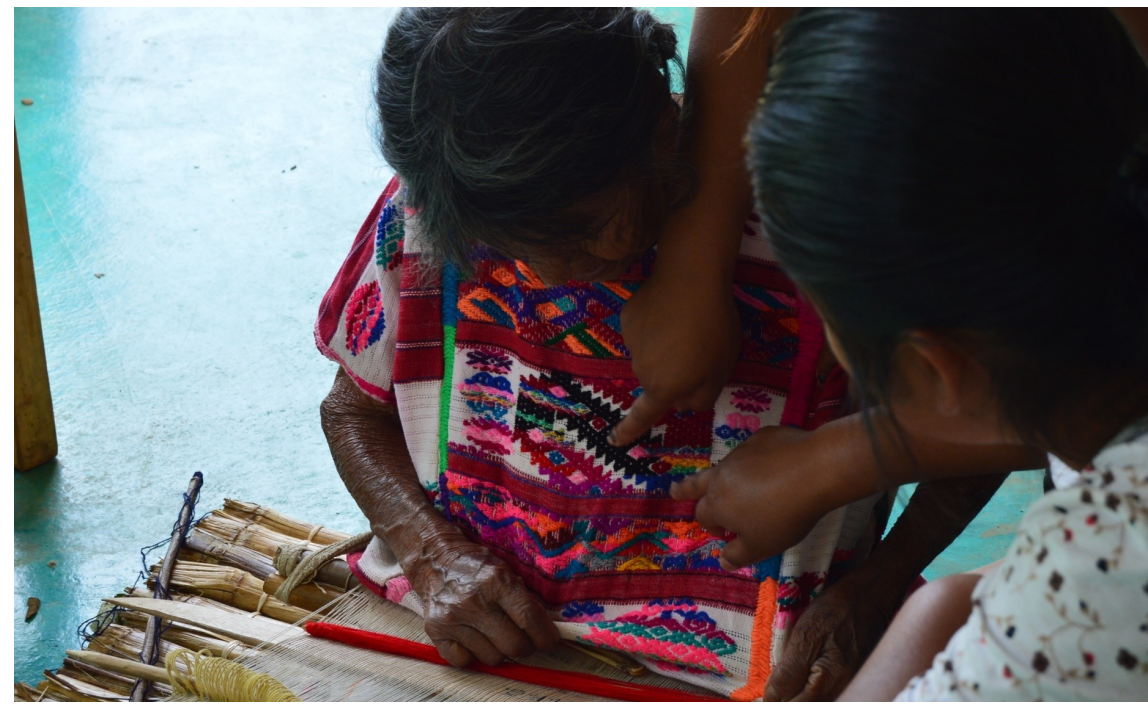

Foto: Estefanía Soto C., 2016

La vieja mujer araña, la mujer maíz, la mujer serpiente, la mujer pensamiento son algunos de los nombres de creadoras poderosas. Para tribus ginecráticas, la mujer está en el centro y nada es más sagrado sin su bendición ni pensamiento". (Lugones, 2007).

\section{Bibliografía}

Bartra, E. (diciembre de 2008). SCielo. Rumiando en torno a lo escrito sobre mujeres y arte popular. Guadalajara, Jalisco, México: Revista Digital, SCielo, Revista de Estudios de Género.

Bartra, E., \& Elías, M. G. (2015). Mujeres, feminismo y arte popular. En E. Bartra, Apuntes sobre feminismo y arte popular (págs. 21 - 29 ). México: Universidad Autónoma Metropolitana, Unidad Xochimilco.

Bartra, R. (2001). El mito del Salvaje. Ciencias, 88 - 96 . Castellanos, A. (s.f.). Asimilación y diferencias de los indios en México.
Castellanos, A. (s.f.). Asimilación y diferencias de los indios en México.

Comisión Nacional para el Desarrollo de los pueblos Indígenas. (2012). Informe de la Consulta Nacional sobre la situación que guardan los derechos de la Mujeres indígenas en sus pueblos y comunidades. México.

Consejo Estatal para la Cultura y las Artes de Hidalgo. (2012). Las Mendoza, familia de artistas. Pachuca, Hidalgo, México.

Díaz, F. A. (1995). Participación femenina, estrategias familiares de vida y jefatura femenina de hogar: los problemas de la jefatura declarada.

Fernández, J. (1972). Estética del Arte Mexicano. UNAM.

Johnson, K., Ávila, A. d., \& Ortíz, D. (2014). Irmagard Weitlander Johnson, una vida dedicada al textil. Oaxaca de Juárez, México: Coorporativa Litográfica de Antequera S.A de C.V. 
Luz María García, e. a. (2006). Las mujeres indígenas de México: su contexto socioeconómico, demográfico y de salud. México.

Mercado Maldonado, A., \& Hernández Oliva, A. V. (2010). El proceso de construcción de la identidad colectiva. Convergencia. Revista de Ciencias Sociales, 229 - 251.

Paalen, I. M. (1970). Historia General del Arte Mexicano. Hermes.

Peña, G. d. (2008). La antropología social y cultural en México.

Ruíz, L. M. (Noviembre de 2012). Memoria Descriptiva de la técnica de bordado textil tradicional de San Bartolo Tijaltepec, Tlaxiaco, Oaxaca. Huajuapán de León, Oaxaca de Juárez, México.

Stavenhagen, R. (1984). Notas sobre la cuestión étnica. Estudios sociológicos. 